\title{
Evaluation of Active Contour-based Techniques toward Bone Segmentation from CT Images
}

\author{
Phan Tran Ho Truc ${ }^{1}$, Yoon Hyuk Kim ${ }^{2}$, Young Koo Lee ${ }^{1}$, Sung Young Lee ${ }^{1}$, and Tae-Seong Kim ${ }^{3}$ \\ ${ }^{1}$ Department of Computer Engineering, Kyung Hee University, Korea \\ ${ }^{2}$ College of Advanced Technology, Kyung Hee University, Korea \\ ${ }^{3}$ Department of Biomedical Engineering, Kyung Hee University, Korea
}

\begin{abstract}
Automatic bone segmentation of Computed Tomography (CT) images is an important step in imageguided surgery where segmentation errors could be critical. Previous attempts include intensity-, edge-, region-, and deformable curve-based approaches [1], but none claims fully satisfactory performance. In this study, we have tested most widely used active contour (AC) -based approaches to segment knee bones from CT imagery, namely the Gradient Vector Flow (GVF) AC, the original geometric AC, the geodesic AC, the GVF fast geometric $\mathrm{AC}$, and the Chan-Vese multiphase AC without edges. Among the techniques, only the Chan-Vese multiphase AC demonstrated satisfactory performance, proving its suitability for bone segmentation from CT images.
\end{abstract}

Keywords- Bone Segmentation, Active Contours, Level Set Methods, CT Images

\section{INTRODUCTION}

Bone segmentation from Computed Tomography (CT) imagery is a critical component in computer-assisted orthopedic surgery, but is a challenging task due to inhomogeneous bone structures, low contrast edges, and overlapping intensity values of bones. There have been a few attempts including intensity-, edge- (no active contours), region-, and deformable curve-based approaches as surveyed in [1]. However, none of them claims fully automatic procedures for bone segmentation. Also, the recent application of geometric active contour (AC) to bone segmentation by $\mathrm{Yao}$ et al. [8] showed very limited performance.

Recently, active contour-based segmentation techniques are being actively researched and developed. In general, there are two types of $\mathrm{AC}$ (or snake) models in literature today: parametric AC's (such as the GVF AC) and geometric $\mathrm{AC}$ 's (such as the original geometric $\mathrm{AC}$, the geodesic $\mathrm{AC}$, the GVF fast geometric AC, and the Chan-Vese multiphase AC without edges). Except for the Chan-Vese snake, all other techniques utilize edge information of images.

A recent survey [1] indicates that the AC models have rarely been applied toward bone segmentation of CT images despite their excellent capabilities for object segmentation. In this study, we have implemented all above mentioned AC approaches and evaluated them by segmenting knee bones from CT data to find the most suitable technique for bone extraction. Our preliminary results indicate that the ChanVese multiphase AC produces excellent segmentation results, proving that fully automatic bone segmentation could be possible.

\section{Methodology}

In this section, we briefly review the AC models we have evaluated in this study.

\section{A. Gradient Vector Flow- based active contour}

The gradient vector flow (GVF) [2] refers to the definition of a slowly-varying, bidirectional external force that helps an active contour reach the object boundaries with a large capture range. One of its distinguished points is the ability to move into the boundary concavities. One can think of this flow field as the optimal direction to be followed to locate the object boundaries.

In order to build up this field, an appropriate edge map function $\mathrm{g}(\mathrm{x}, \mathrm{y})$ having larger values near the image edges is first chosen. To this end, while any gray-level edge map defined in the image processing literature could be a good candidate, a Gaussian-derived function on the image gradient is usually considered for its smooth characteristic:

$$
g(x, y)=1-\frac{1}{\sqrt{2 \pi} \sigma_{E}} e^{-\frac{\left|\nabla\left(G_{\sigma}^{*}\right)(x, y)\right|^{2}}{2 \sigma_{E}^{2}}}
$$

where $\sigma_{\mathrm{E}}$ is variance and $\left[\mathrm{G}_{\sigma} * \mathrm{I}\right]$ is the convolution output of the input image with a Gaussian kernel.

The GVF [2] is defined as a two-dimensional vector field $[\mathrm{u}(\mathrm{x}, \mathrm{y}), \mathrm{v}(\mathrm{x}, \mathrm{y})]$ that minimizes the following energy functional

$$
E(u, v)=\iint_{\Omega} \mu\left(u_{x}^{2}+u_{y}^{2}+v_{x}^{2}+v_{y}^{2}\right)+|\nabla g|^{2}|(u, v)-\nabla g|^{2} d x d y(2)
$$

where $\mathrm{u}_{\mathrm{x}}, \mathrm{u}_{\mathrm{y}}, \mathrm{v}_{\mathrm{x}}$ and $\mathrm{v}_{\mathrm{y}}$ are the spatial derivatives of the field and $\mu$ a noise-control parameter.

This functional keeps the GVF field nearly same as the gradient of the edge map, $\nabla \mathrm{g}$, in the neighborhood of the boundaries, where $|\nabla g|$ is large. At the same time, the field still have significant values in the homogeneous regions, where $|\nabla \mathrm{g}|$ gets close to 0 , via a diffusion process.

One can optimize $[\mathrm{u}(\mathrm{x}, \mathrm{y}), \mathrm{v}(\mathrm{x}, \mathrm{y})]$ using the gradient descent method and the calculus of variations

$$
\frac{d u}{d t}=\mu \nabla^{2} u-\left(u-g_{x}\right)|\nabla g|^{2} ; \frac{d v}{d t}=\mu \nabla^{2} v-\left(v-g_{y}\right)|\nabla g|^{2}
$$

where $g_{x}, g_{y}$ are the spatial derivatives of $g$. 
In [2], a parametric $\mathrm{AC}$ using the normalized GVF (NGVF) $\left[\hat{u}=u / \sqrt{u^{2}+v^{2}}, \hat{v}=v / \sqrt{u^{2}+v^{2}}\right]$ was proposed for boundary extraction in the following way,

$$
C_{t}(p)=\alpha \frac{\partial^{2} C}{\partial p^{2}}(p)-\beta \frac{\partial^{4} C}{\partial p^{4}}(p)+[\hat{u}(p), \hat{v}(p)]
$$

where $C(p)=[x(p), y(p)]:[0,1] \rightarrow R^{2}$ is the parameterized curve, $\alpha$ and $\beta$ the adjustment constants.

Such a flow depends on the parameterization of the curve and cannot topologically change to track multiple objects. Also it involves the second and fourth order derivatives that are difficult to estimate. It is, however, relatively free of the initial conditions and can deal with concave regions. Moreover, GVF AC application in bone segmentation, where the multiple-object extraction is not necessary, provides the advantage of fast convergence in comparison with others.

\section{B. Geometric AC and geodesic AC}

Geometric active contours $[4,5]$ are based on the theory of curve evolution [9] and the level set method [10]. In this framework, curves are implicitly represented via a Lipschitz function and their evolutions are performed using only geometric measures, making themselves independent of the curves' parameterization.

Toward the construction of the flow, let define $\phi(x, t)$ as a scalar Lipschitz function whose zero level set represents the geometric active contour. The original geometric active contour flow evolves $\phi$ according to

$$
\phi_{t}=g\left(\kappa+V_{0}\right)|\nabla \phi|
$$

where $\kappa$ is the Euclidean curvature, $V_{0}$ a constant, and $g \equiv g(x)=e^{-\frac{1}{2}\left|G_{\sigma}(x) * I(x)\right|^{2}}$ an edge-based function.

In Eq. (5), the curvature $\kappa$-based flow has the properties of smoothing the curve, while $\mathrm{V}_{0}$ shrinks or expands the contour along its normal direction at a constant velocity. The product $\mathrm{g}\left(\kappa+\mathrm{V}_{0}\right)$ determines the overall evolution speed of level sets of $\phi(x, t)$. At the same time, the main use of $g$ has the effect of stopping the curve when it reaches to the object boundaries.

In contrast with parametric AC's, the geometric flow is topology independent and thus allows the $\mathrm{AC}$ to detect multiple objects. This scheme works well in general for objects that have good contrast. In cases where there are high variations of gradient as well as gaps along the edge, this contour, however, tends to pass through the object boundary. To overcome this limitation, the following evolution flow, called the geodesic AC flow, was proposed in [6, 7, and 13] and can be expressed as

$$
\phi_{t}=g\left(\kappa+V_{0}\right)|\nabla \phi|+\nabla g \cdot \nabla \phi
$$

Comparing with the old model given in Eq. (5), we see that the extra stopping term $(\nabla \mathrm{g} \cdot \nabla \phi)$ is used to increase the attraction of the evolving contour towards the boundary, especially when it has different gradient values and/or gaps.

\section{GVF fast geometric AC}

Inspired from the observations that the NGVF consists of the direction to be followed to reach the object boundaries and that evolving the contour in the direction of its normal is a main characteristic of the geometric active contour flow, Paragios et al. [3] proposed an integration of NGVF into the geometric $\mathrm{AC}$ :

$$
C_{t}(p)=g \cdot(\beta \kappa+(1-|H(p)|)[\hat{u}, \hat{v}] \cdot N(p)+H(p)) N(p)
$$

The level set implementation of this flow can be expressed as

$$
\phi_{t}(p)=g \cdot((H(p)+\beta \kappa)|\nabla \phi(p)|-(1-|H(p)|)[\hat{u}, \hat{v}] \cdot \nabla \phi(p))
$$

where $\mathrm{N}(\mathrm{p})$ is the inward Euclidean normal of the curve and $H(p)=\operatorname{sign}([\hat{u}, \hat{v}] \cdot N(p)) e^{-\delta[\hat{u}, \hat{v}] \cdot N(p) \mid}$, with $\delta$ a scale factor, has significant values when the normal and the NGVF are close to orthogonal.

In the above flow, the use of $\kappa$ has the effect of regularizing the propagation, and the use of $(1-|\mathrm{H}(\mathrm{p})|)(\mathrm{NGVF} \cdot \nabla \phi)$ has the effect of a bidirectional flow that moves the curve towards object boundaries from either sides, while the remaining term $\mathrm{H}(\mathrm{p})|\nabla \phi|$ has the effect of an adaptive balloon force used to determine the evolution when the bidirectional flow term becomes inactive. Similar to that of the geometric $\mathrm{AC}$, the overall speed of this curve evolution is coupled with the edge-driven information via a stopping term $\mathrm{g}$.

\section{Chan-Vese multiphase AC without edges}

Chan and Vese proposed in [11] an alternative form of $\mathrm{AC}$, called AC without edges, based on the Mumford and Shah functional for segmentation [14] and level set framework [10]. Unlike other level-set-based AC's which rely much on the gradient of the image as the stopping term and thus has unsatisfactory performance in noisy images, the Chan and Vese model does not use the edge information but utilize the difference between the regions inside and outside of the curve.

To this end, a signed distance function $\phi(\mathrm{x}, \mathrm{y})$ is used to represent the curve $\mathrm{C}$ such that

$$
\left\{\begin{array}{c}
C=\{(x, y): \phi(x, y)=0\} \\
\text { inside }(C)=\{(x, y): \phi(x, y)>0\} \\
\text { outside }(C)=\{(x, y): \phi(x, y)<0\}
\end{array}\right.
$$

In this case, the image is assumed to be consisted of two areas with approximately piecewise-constant intensities, of different value $c_{\text {in }}$ and $c_{\text {out }}$. The arbitrarily initialized active contour $\mathrm{C}$ will be placed exactly on the boundary of these two regions if both differences between $\mathrm{c}_{\text {in }}$ and image intensities inside $\mathrm{C}$, and between $\mathrm{c}_{\text {out }}$ and image intensities outside $\mathrm{C}$ are minimized. Those amounts of dissimilarities are therefore playing the role of an external evolution force in the level set formulation of this model:

$$
\frac{\partial \phi}{\partial t}=|\nabla \phi|\left[v \cdot \operatorname{div}\left(\frac{\nabla \phi}{|\nabla \phi|}\right)-\left(u_{0}-c_{1}\right)^{2}+\left(u_{0}-c_{2}\right)^{2}\right]
$$


where $\mathrm{u}_{0}(\mathrm{x}, \mathrm{y})$ is the given image, $\mathrm{v}$ a smoothing constant and $c_{1}$ and $c_{2}$ respectively the average intensities inside and outside $\mathrm{C}$ at the current instant.

This flow evolves the AC, looking for a two-phase segmentation of the image, given by $\mathrm{u}(\mathrm{x}, \mathrm{y})=\mathrm{c}_{\mathrm{in}} \mathrm{H}[\phi(\mathrm{x}, \mathrm{y})]+$ $\mathrm{c}_{\text {out }}(1-\mathrm{H}[\phi(\mathrm{x}, \mathrm{y})])$, where $\mathrm{H}$ is the Heaviside function:

$$
H(z)= \begin{cases}1 & z \geq 0, \\ 0 & z<0 .\end{cases}
$$

The main advantages of the Chan and Vese snake over other active contour models are 1) it automatically detects interior contours and 2) the initial curve can be placed anywhere in the image.

For multiphase case, we can write the illustration formulations for four phases or classes (and therefore using the two level sets functions $\left.\phi_{1}, \phi_{2}\right)$ as

$$
\begin{gathered}
\frac{\partial \phi_{1}}{\partial t}=\left|\nabla \phi_{1}\right|\left\{v \cdot \operatorname{div}\left(\frac{\nabla \phi_{1}}{\left|\nabla \phi_{1}\right|}\right)-\left[\left(u_{0}-c_{11}\right)^{2}-\left(u_{0}-c_{01}\right)^{2}\right] H\left(\phi_{2}\right)\right. \\
\left.-\left[\left(u_{0}-c_{10}\right)^{2}-\left(u_{0}-c_{00}\right)^{2}\right]\left(1-H\left(\phi_{2}\right)\right)\right\} \\
\frac{\partial \phi_{2}}{\partial t}=\left|\nabla \phi_{2}\right|\left\{v \cdot \operatorname{div}\left(\frac{\nabla \phi_{2}}{\left|\nabla \phi_{2}\right|}\right)-\left[\left(u_{0}-c_{11}\right)^{2}-\left(u_{0}-c_{10}\right)^{2}\right] H\left(\phi_{1}\right)\right. \\
\left.-\left[\left(u_{0}-c_{01}\right)^{2}-\left(u_{0}-c_{00}\right)^{2}\right]\left(1-H\left(\phi_{1}\right)\right)\right\}
\end{gathered}
$$

where $\mathrm{c}_{11}(\phi)=$ average $\left(\mathrm{u}_{0}\right)$ in $\left\{(\mathrm{x}, \mathrm{y}): \phi_{1}(\mathrm{x}, \mathrm{y})>0, \phi_{2}(\mathrm{x}, \mathrm{y})>0\right\}$

$\mathrm{c}_{10}(\phi)=\operatorname{average}\left(\mathrm{u}_{0}\right)$ in $\left\{(\mathrm{x}, \mathrm{y}): \phi_{1}(\mathrm{x}, \mathrm{y})>0, \phi_{2}(\mathrm{x}, \mathrm{y})<0\right\}$

$\mathrm{c}_{01}(\phi)=\operatorname{average}\left(\mathrm{u}_{0}\right)$ in $\left\{(\mathrm{x}, \mathrm{y}): \phi_{1}(\mathrm{x}, \mathrm{y})<0, \phi_{2}(\mathrm{x}, \mathrm{y})>0\right\}$

$\mathrm{c}_{00}(\phi)=\operatorname{average}\left(\mathrm{u}_{0}\right)$ in $\left\{(\mathrm{x}, \mathrm{y}): \phi_{1}(\mathrm{x}, \mathrm{y})<0, \phi_{2}(\mathrm{x}, \mathrm{y})<0\right\}$

\section{EXPERIMENT RESULTS}

To evaluate the suitability of AC's toward bone segmentation, we applied the mentioned five techniques to a set of $16 \mathrm{CT}$ image slices covering knee regions. We considered the segmentation is satisfactory if the AC correctly find the bone boundaries visually. However, unsatisfactory, if the AC does not converge to the correct boundaries.

Figs. 1-4 show the segmentation results obtained using the GVF, the GVF fast geometric, the original geometric, and the geodesic AC respectively. In these cases, the initial contours were placed inside the bone regions because the outside-initialized contours tend to stop at the outter boundary and can never evolve towards the inner one as expected.

The lower left part of the GVF snake after a certain number of iterations could not propagate anymore because the NGVF in that area is close to orthogonal to the inward normal of the curve as shown in Fig. 1 (c). This is plausible when dealing with noisy images where some noise can be accidentally considered as line segments. To solve this problem, the Gaussian-derived edge map function should be more stringent, but it, in turn, smoothes the edges as well. In this situation, the balloon force in the GVF fast geometric AC model in Eq. (8) can help to overcome this limitation.
However, as shown in Fig. 2 (c), it tends to pass through and thus cannot stop at the desired but weak edges.

On the other hand, although the geometric AC model in Eq. (5) can deal with multiple objects, it suffers from the same problem of the GVF fast geometric AC. Comparing Figs. 3 (c) and 4 (c), we can see that the geodesic AC with the extra stopping term that pulls back the boundarypassing-through contour still does not yield satisfactory performance in the blurred bone boundaries of CT images.

In Fig. 5, we show that the Chan and Vese multiphase (i.e., four phases with two level set functions) AC without edges can successfully find bone boundaries with high gradient variations. With the robust initialization as shown in Fig. 5 (a), where the AC evolution is demonstrated, the AC finds correct boundaries of all given slices. This model works well in this case because it does not deal with edge information and does not depends on the initial conditions. The only factor determining its performance is the difference of intensity inside and outside the bone regions.
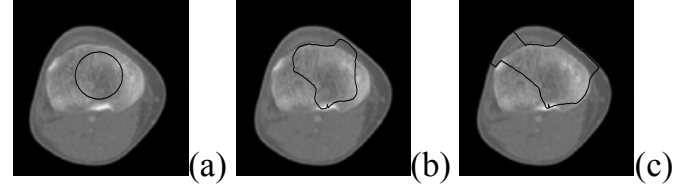

Fig. 1. GVF AC evolution: (a) - (c) $\mathrm{t}=0,25$, and 125 iterations
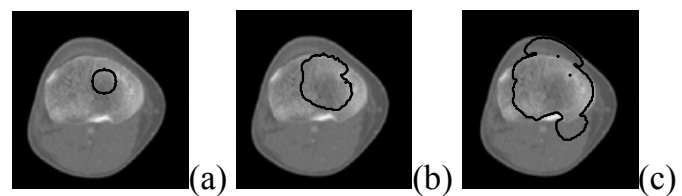

Fig. 2. GVF fast geometric AC evolution: (a) - (c) $\mathrm{t}=0,150$, and $300 \mathrm{sec}$
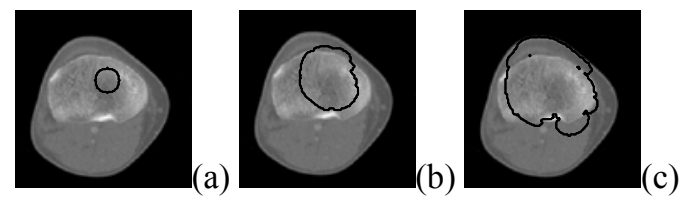

Fig. 3. Geometric AC evolution: (a) - (c) $\mathrm{t}=0,25$, and $50 \mathrm{sec}$
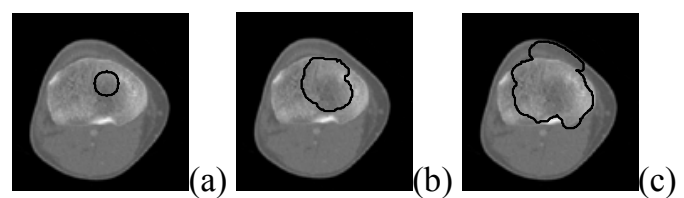

Fig. 4. Geodesic AC evolution: (a) - (c) $\mathrm{t}=0$, 70, and $140 \mathrm{sec}$

\section{DISCUSSION AND CONCLUSION}

We have presented the evaluation results of various active contour-based techniques toward knee bone segmentation from CT images. The results show that most edgebased AC's failed to detect correct boundaries of the bone, but the Chan-Vese multiphase AC without edges succeeded. The evaluation results indicate that the edge-based AC's suffer from high sensitivity to noise, low-contrast bounda- 
ries, and difficulty in getting distinct bone edges. However, the $\mathrm{AC}$ without edges showed robustness in segmenting the bone correctly with some advantages such as robustness in curve initialization, automatic detection of inner contours, and ability to represent different objects and their boundaries. We consider that the region-based $\mathrm{AC}$ without edges could be a preferred choice in the automatic bone segmentation work.

\section{ACKNOWLedgment}

This work was supported by grant No. (R01-2006-00011209-0) from the Basic Research Program of the Korea Science \& Engineering Foundation.

\section{REFERENCES}

1. L. Wang, M. Greenspan and R. Ellis (2006). Validation of Bone Segmentation and Improved 3-D Registration using Contour Coherence in CT Data. IEEE Trans. Med. Imag., 25: 324-334.

2. C. Xu and J. Prince (1998). Snakes, Shapes, and Gradient Vector Flow. IEEE Trans. Image Processing, vol. 7, pp. 359-369.

3. N. Paragios, O.M-Gottardo and V. Ramesh (2004). Gradient Vector Flow Fast Geometric Active Contours. IEEE Trans. Patt. Anal. Mach. Intell., vol. 26, pp. 402-407

4. Caselles, F. Catte, T. Coll, and F. Dibos (1993). A geometric model for active contours. Numerische Mathematik, 66:1-31.

5. R. Malladi, J. A. Sethian, and B. C. Vemuri (1995). Shape modeling with front propagation: a level set approach. IEEE Trans. Patt. Anal. Mach. Intell., 17(2):158-175.
6. V. Caselles, R. Kimmel, and G. Sapiro (1995). Geodesic active contours. Proc. Int. Conf. Comp. Vis., pp. 694-699.

7. A. Yezzi, S. Kichenassamy, A. Kumar, P. Olver, and A. Tannenbaum (1997). A geometric snake model for segmentation of medical imagery. IEEE Trans. Med. Imag., 16:199-209.

8. W. Yao, P. Abolmaesumi, M. Greenspan and R. Ellis (2005). An estimation/correction algorithm for detecting bone edges in CT images. IEEE Trans. Med. Imag., 24:997-1010

9. G. Sapiro and A. Tannenbaum (1993). Affine invariant scale-space. Int. J. Comp. Vis., 11(1):25-44.

10. S. Osher and J. A. Sethian (1998). Fronts propagating with curvaturedependent speed: algorithms based on Hamilton-Jacobi formulations. J. Comp. Physics, 79:12-49.

11. T. Chan and L. Vese (2001). Active contours without edges. IEEE Trans. IP, vol. 10, pp. 266-277

12. L. Vese and T. Chan (2002). A multiphase level set framework for Image Segmentation using Mumford and Shah Model. Int. J. Comp. Vis., 50(3): 271-293.

13. V. Caselles, R. Kimmel, and G. Sapiro (1997). Geodesic active contours. Int. J. Comp. Vis., 22:61-79

14. D. Mumford and J. Shah (1989). Optimal approximation by piecewise smooth functions and associated variational problems. Commun. Pure Appl. Math, 42: 577-685

Address of the corresponding author:

Author: Tae-Seong Kim, Ph.D

Institute: Kyung Hee University

Street: 1 Seocheon-dong, Giheung-gu, Yongin-si,

City: Gyeonggi-do

Country: Republic of Korea 446-701

Email: tskim@khu.ac.kr (a)

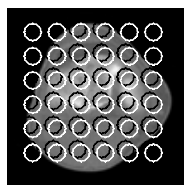

Oiginalimage th

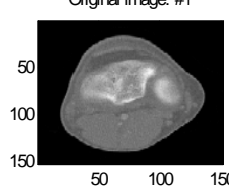

Original image: \#3

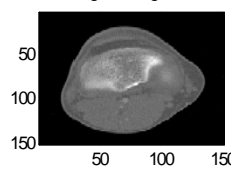

(b)
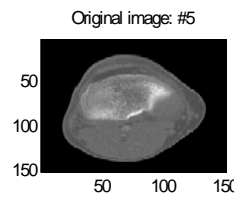

Original image: \#7
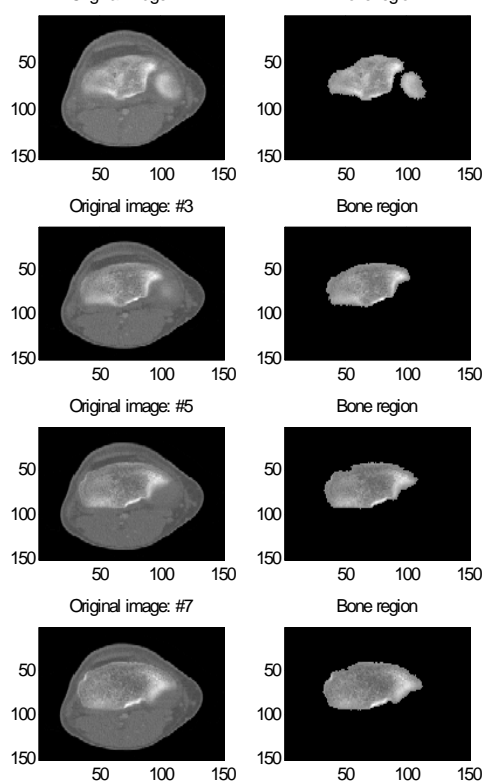

Bone region

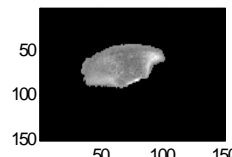

Bone region

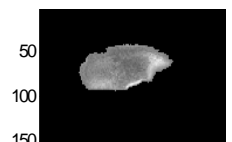

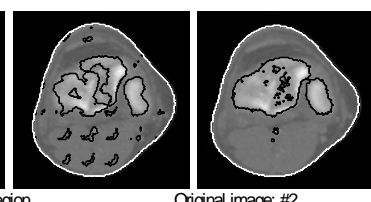

Original image: \#2
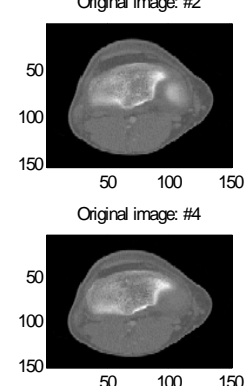

Original image: \#6

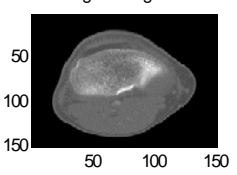

Original image: \#8
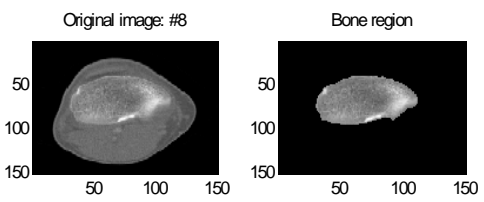

Fig. 5. Segmentation results using the Chan - Vese multiphase AC without edges: a) Initial contours and their evolution. b) CT images in Column 1 and 3 and segmented bone regions in Column 2 and 4. 